R. J. Cohen and W. T. Sullivan, III, eds.

\title{
Educating the Public about Light Pollution
}

\author{
Syuzo Isobe and Shiomi Hamamura \\ National Astronomical Observatory, Mitaka, Tokyo, Japan \\ Christopher D. Elvidge \\ NOAA National Geophysical Data Center, Boulder, Colorado, U.S.A.
}

\begin{abstract}
Using low-gain DMSP data, we obtained absolute values of light energy loss ejected to space from different cities. Showing these data to local people in each city encourages them to try to reduce light energy loss. This educational approach is very effective in reducing light pollution.
\end{abstract}

\section{Introduction}

It is true that the public need and enjoy illuminating light. However, outdoor lighting is producing light pollution. We professional and amateur astronomers have a tendency to request light reduction from the public with arguments that star fields are beautiful and that scientific outputs are important for humanbeings. Sometimes we succeed in making the public turn down the lights, especially for astronomically interesting events such as Comet Hyakutake, Comet Hale-Bopp and the Leonid showers.

It is also true that the fraction of people enjoying astronomical observations and star-watching is not large, that is, only one tenth or one hundredth of the total population. In order to get the support of the majority of people, we have to develop a clear way to educate the public. Since we have the DMSP (Defense Meteorological Satellite Program) data, we are trying to develop a new way to reduce light pollution.

\section{Energy Loss}

Electrical engineering has been much developed in the 20th century, especially in these last decades, during which people have enjoyed a much brighter nighttime environment. However, the public and also lighting designers want to have lighting fixtures which are well decorated, especially in day time. Then they most easily use lighting fixtures which eject a large fraction of light towards the sky (Kawakami \& Isobe 1998), and this light becomes energy loss.

Light pollution of astronomical observations is mainly produced by light ejected in directions with elevation angles of $0^{\circ}$ to $45^{\circ}$. As an example, such an effect was studied by Osman et al. (2001) for the case of Kottamia Observatory 
in Egypt. City light ejected directly in near-zenith directions is only energy loss but does not create much light pollution of nearby observatories.

\section{DMSP Data}

The US Air Force started the DMSP in 1972 and made continuous observations using a series of satellites. However, after the US Air Force had used those data, they had no interest in keeping the data in digital form and just kept photographic prints. Using those prints Sullivan (1991) produced a famous map of Earth at Night and later Nakayama (1992) read the brightness distributions of all the photographic prints into a computer, corrected the projection effects and obtained a better map of the Earth at Night.

Fortunately, since 1993 the National Geographic Data Center receive digital data from the US Air Force and keep all the data in the form of 8-mm tapes. Through some communications with a leader on this programme, Dr. Elvidge of NGDC, we also get the data interesting for us as a collaborative project with NGDC.

The DMSP data from 1993 to 1996 were obtained using a high-gain mode which can detect light levels within the range $8 \times 10^{-11} \mathrm{~W} / \mathrm{cm}^{2} / \mathrm{sr} / \mu \mathrm{m}$ to $7 \times 10^{-9}$ $\mathrm{W} / \mathrm{cm}^{2} / \mathrm{sr} / \mu \mathrm{m}$ (Elvidge et al. 1999), but a large fraction of the area of big cities was usually saturated. Therefore, we cannot measure absolute values of light energy detected. However, we can still obtain a reasonable result for small cities and we have demonstrated increases of light energy loss from 1993 to 1997 in the cities of Akita, Shizuoka, Hiroshima, Matsuyama, and Tokushima in Japan (Isobe \& Hamamura 2000).

By a strong request of Dr. C. Elvidge, the US Air Force made several observations with low gain. Gain number is different from time to time, but in most cases we could get non-saturated data, except for the central parts of very big cites such as Tokyo.

Table 1 shows all the results that we could reduce to date. Although we have a much larger amount of data, we have reduced only a small part of them because of a shortage of manpower. However, we will extend our efforts. Figures 1 and 2 show two maps of the brightness distribution. Further results of this ongoing work, tables and a picture gallery, can be viewed at the following web site, http://neowg.mtk.nao.ac.jp/ .

Fortunately, in 1999 the US Air Force plan to observe with low-gain much more frequently and then we will be able to compare values of light energy loss at different years.

\section{Discussion}

In Table 1 , column 2, the observed value is just for observed total intensity in each area. Light energy loss per year is estimated assuming that the amount of energy lost to space is constant for 10 hours per night for each night of one year. Then light energy loss $/ \mathrm{km}^{2} /$ year is calculated. Since the dynamic range of the detector is not so large, the resulted light energy loss $/ \mathrm{km}^{2} /$ year estimated for most of the cities has values spanning only a factor of 20 . 


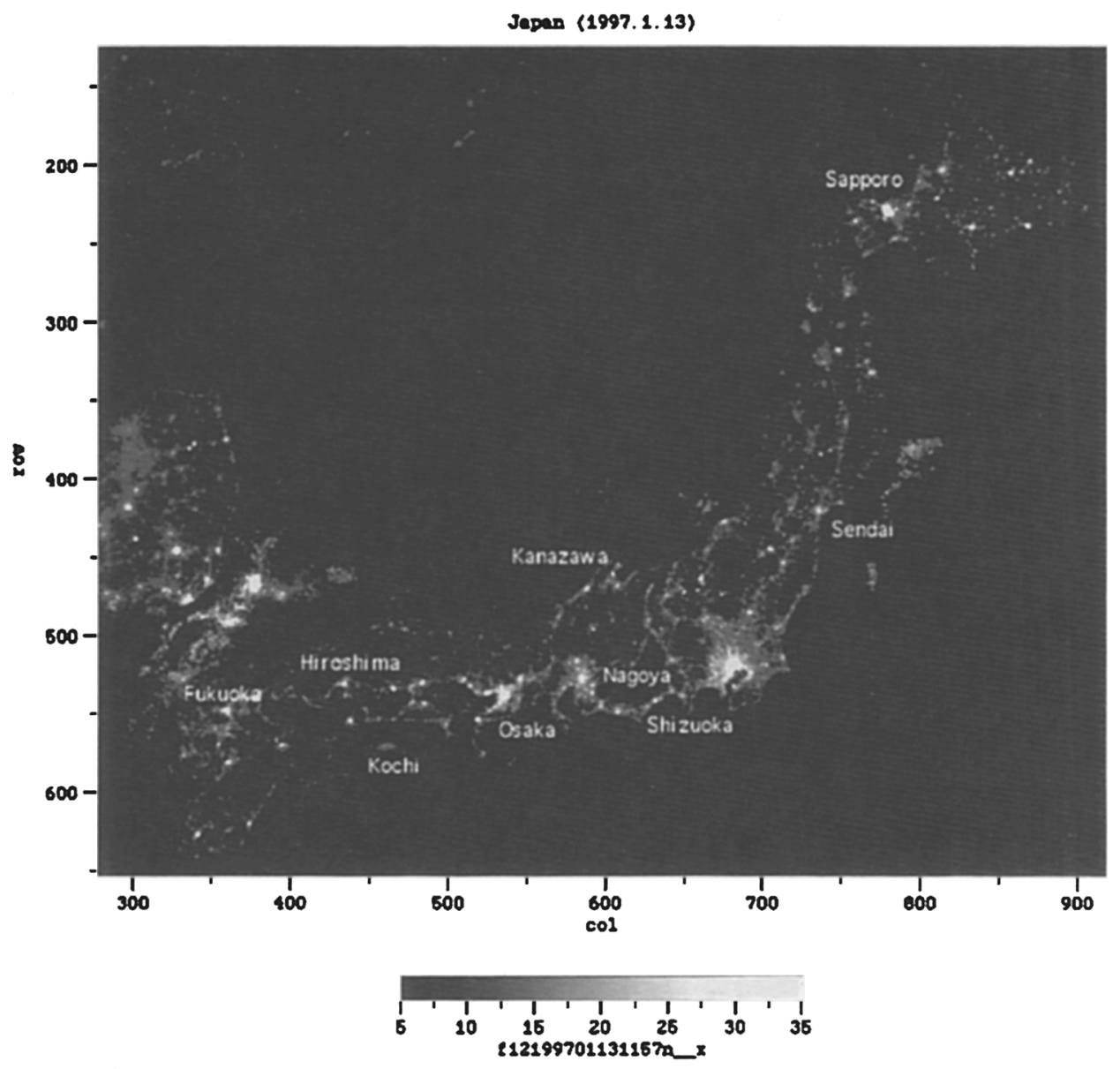

Figure 1. Brightness distribution of Japan on 13 January 1997.

There are fairly high values for cities in Canada, which may be caused by light reflection from snow on the roads. We see a similar effect for the Japanese city of Sapporo. To escape this snow effect, we should use low-gain data obtained in a season other than winter. However, only winter data are available in 1997 and therefore we have to wait to make a definite conclusion.

Although our data have still a problem to be resolved, we have now a fairly large number of light energy loss values for cities. In Japan, the Environmental Agency set a guideline to protect light pollution and assigned six cities to work on this. Then, if some city tries to reduce upward light, we can detect a decrease of light energy loss. Local people can see that their effort is directly linked to reduced energy loss and get a triggering motivation to consider the conservation of energy for future generations.

This kind of work is not a direct method to reduce a light pollution, but a certain method for making it happen. 
Table 1. Energy detected by the DMSP and values estimated from it.

\begin{tabular}{|c|c|c|c|c|}
\hline & $\begin{array}{c}\text { Observed } \\
\text { Value } \\
\left(10^{-8} \mathrm{~W} / \mathrm{cm}^{2} / \mathrm{st} / \mu \mathrm{m}\right)\end{array}$ & $\begin{array}{l}\text { Light Energy } \\
\text { Loss/Year } \\
\left(10^{6} \text { kWh }\right)\end{array}$ & $\begin{array}{c}\text { Area } \\
\left(\mathbf{k m}^{2}\right)\end{array}$ & $\begin{array}{r}\text { Light Energy } \\
\text { Loss/Area/Year } \\
\left(10^{6} \mathbf{k W h} / \mathbf{k m}^{2}\right)\end{array}$ \\
\hline \multicolumn{5}{|l|}{ Japan (1997.1.13) } \\
\hline $\begin{array}{l}\text { Sapporo } \\
\text { Sendai }\end{array}$ & $2.47 \times 10^{3}$ & 14.8 & 1046 & $\begin{array}{l}1.41 \times 10^{-2} \\
9.57 \times 10^{-3}\end{array}$ \\
\hline $\begin{array}{l}\text { Sendai } \\
\text { Kanazawa }\end{array}$ & $7.40 \times 10^{2}$ & 4.43 & $\begin{array}{l}463 \\
543\end{array}$ & $\begin{array}{l}9.57 \times 10^{-3} \\
5.71 \times 10^{-3}\end{array}$ \\
\hline $\begin{array}{l}\text { Kanazawa } \\
\text { Shizuoka }\end{array}$ & $\begin{array}{l}5.18 \times 10^{2} \\
4.56 \times 10^{2}\end{array}$ & $\begin{array}{l}3.10 \\
2.73\end{array}$ & $\begin{array}{l}543 \\
528\end{array}$ & $\begin{array}{l}5.71 \times 10^{-3} \\
5.17 \times 10^{-3}\end{array}$ \\
\hline Nagoya & $3.83 \times 10^{3}$ & 22.9 & 1519 & $1.51 \times 10^{-2}$ \\
\hline Osaka & $5.85 \times 10^{3}$ & 35.1 & 1896 & $1.85 \times 10^{-2}$ \\
\hline Hiroshima & $8.72 \times 10^{2}$ & 5.22 & 1001 & $5.21 \times 10^{-3}$ \\
\hline Kochi & $2.39 \times 10^{2}$ & 1.43 & 729 & $1.96 \times 10^{-3}$ \\
\hline Fukuoka & $1.56 \times 10^{3}$ & 9.35 & 1026 & $9.11 \times 10^{-3}$ \\
\hline \multicolumn{5}{|l|}{ Korea $(1997.2 .27)$} \\
\hline Seoul & $7.07 \times 10^{3}$ & 42.4 & 2266 & $1.87 \times 10^{-2}$ \\
\hline Pusan & $1.49 \times 10^{3}$ & 8.96 & 910 & $9.85 \times 10^{-3}$ \\
\hline Pyongyang & 2.38 & 0.0143 & 133 & $1.08 \times 10^{-4}$ \\
\hline \multicolumn{5}{|l|}{ Europe (1997.1.13) } \\
\hline London & $4.84 \times 10^{3}$ & 29.0 & 2030 & $1.43 \times 10^{-2}$ \\
\hline Amsterdam & $1.07 \times 10^{3}$ & 6.43 & 367 & $1.75 \times 10^{-2}$ \\
\hline Leiden & $2.16 \times 10^{2}$ & 1.29 & 138 & $9.35 \times 10^{-3}$ \\
\hline Bruxelles & $9.64 \times 10^{2}$ & 5.78 & 536 & $1.08 \times 10^{-2}$ \\
\hline Paris & $6.33 \times 10^{3}$ & 37.9 & 2091 & $1.81 \times 10^{-2}$ \\
\hline \multicolumn{5}{|l|}{ Europe (1997.2.3) } \\
\hline Wein & $1.20 \times 10^{3}$ & 7.19 & 1080 & $6.66 \times 10^{-3}$ \\
\hline Budapest & $1.58 \times 10^{3}$ & 9.44 & 1331 & $7.09 \times 10^{-3}$ \\
\hline Praha & $1.26 \times 10^{3}$ & 7.55 & 1020 & $7.40 \times 10^{-3}$ \\
\hline Bratislava & $4.25 \times 10^{3}$ & 2.55 & 389 & $6.56 \times 10^{-3}$ \\
\hline Warszawa & $1.47 \times 10^{3}$ & 8.81 & 950 & $9.27 \times 10^{-3}$ \\
\hline Dresden & $9.23 \times 10^{2}$ & 5.53 & 1162 & $4.76 \times 10^{-3}$ \\
\hline Brno & $4.02 \times 10^{2}$ & 2.41 & 384 & $6.28 \times 10^{-3}$ \\
\hline Krakow & $7.35 \times 10^{2}$ & 4.40 & 592 & $7.43 \times 10^{-3}$ \\
\hline Milano & $2.32 \times 10^{3}$ & 13.9 & 1434 & $9.69 \times 10^{-3}$ \\
\hline Zagreb & $4.78 \times 10^{2}$ & 2.86 & 380 & $7.53 \times 10^{-3}$ \\
\hline \multicolumn{5}{|l|}{ Greece (1997.2.5) } \\
\hline Athinai & $2.49 \times 10^{3}$ & 14.9 & 1837 & $8.11 \times 10^{-3}$ \\
\hline Tessaloniki & $6.67 \times 10^{2}$ & 4.00 & 711 & $5.63 \times 10^{-3}$ \\
\hline Larisa & $1.13 \times 10^{2}$ & 0.674 & 219 & $3.08 \times 10^{-3}$ \\
\hline Volos & $1.25 \times 10^{2}$ & 0.749 & 210 & $3.57 \times 10^{-3}$ \\
\hline Lamia & 65.6 & 0.393 & 148 & $2.66 \times 10^{-3}$ \\
\hline Iraklion & $1.06 \times 10^{2}$ & 0.637 & 273 & $2.33 \times 10^{-3}$ \\
\hline \multicolumn{5}{|c|}{ Middle East (1997.1.9) } \\
\hline Tel Aviv-Yafo & $1.72 \times 10^{3}$ & 10.3 & 813 & $1.27 \times 10^{-2}$ \\
\hline Jerusalem & $7.40 \times 10^{2}$ & 4.43 & 511 & $8.67 \times 10^{-3}$ \\
\hline Amman & $8.77 \times 10^{2}$ & 5.25 & 478 & $1.10 \times 10^{-2}$ \\
\hline Haifa & $5.53 \times 10^{2}$ & 3.31 & 253 & $1.31 \times 10^{-2}$ \\
\hline Damascus & $4.98 \times 10^{2}$ & 2.98 & 320 & $9.31 \times 10^{-3}$ \\
\hline Beirut & $6.48 \times 10^{2}$ & 3.88 & 464 & $8.36 \times 10^{-3}$ \\
\hline Baghdad & $9.39 \times 10^{2}$ & 5.62 & 1510 & $3.72 \times 10^{-3}$ \\
\hline \multicolumn{5}{|l|}{ Egypt (1997.2.5) } \\
\hline Cairo & $4.51 \times 10^{3}$ & 27.0 & 1968 & $1.37 \times 10^{-2}$ \\
\hline Alexandria & $6.52 \times 10^{2}$ & 3.90 & 818 & $4.77 \times 10^{-3}$ \\
\hline Ismailiya & $2.88 \times 10^{2}$ & 1.73 & 273 & $6.34 \times 10^{-3}$ \\
\hline Suez & $3.38 \times 10^{2}$ & 2.02 & 264 & $7.65 \times 10^{-3}$ \\
\hline
\end{tabular}


Table 1. continued

\begin{tabular}{|c|c|c|c|c|}
\hline & $\begin{array}{c}\text { Observed } \\
\text { Value } \\
\left(10^{-8} \mathrm{~W} / \mathrm{cm}^{2} / \mathrm{st} / \mu \mathrm{m}\right)\end{array}$ & $\begin{array}{l}\text { Light Energy } \\
\text { Loss/Year } \\
\left(10^{6} \mathrm{kWh}\right)\end{array}$ & $\begin{array}{r}\text { Area } \\
\left(\mathbf{k m}^{2}\right)\end{array}$ & $\begin{array}{r}\text { Light Energy } \\
\text { Loss/Area/Year } \\
\left(10^{6} \mathbf{k W h} / \mathbf{k m}^{2}\right)\end{array}$ \\
\hline \multicolumn{5}{|l|}{ Canada (1997.1.12) } \\
\hline Quebec & $6.13 \times 10^{3}$ & 36.7 & 1767 & $2.08 \times 10^{-2}$ \\
\hline Trois Riviere & $1.23 \times 10^{3}$ & 7.37 & 36 & 0.205 \\
\hline Montreal & $2.32 \times 10^{4}$ & 139 & 4039 & $3.44 \times 10^{-2}$ \\
\hline Ottawa & $5.44 \times 10^{3}$ & 32.6 & 1612 & $2.02 \times 10^{-2}$ \\
\hline Toronto & $2.29 \times 10^{4}$ & 137 & 4330 & $3.16 \times 10^{-2}$ \\
\hline Sudbury & $1.41 \times 10^{3}$ & 8.45 & 603 & $1.40 \times 10^{-2}$ \\
\hline Chicoutimi & $1.28 \times 10^{3}$ & 7.65 & 400 & $1.91 \times 10^{-2}$ \\
\hline Calgary & $1.39 \times 10^{4}$ & 83.4 & 1901 & $4.39 \times 10^{-2}$ \\
\hline Edmonton & $9.83 \times 10^{3}$ & 58.9 & 1819 & $3.24 \times 10^{-2}$ \\
\hline \multicolumn{5}{|l|}{ U.S.A. (1997.2.4) } \\
\hline New York (Long Is.) & $2.26 \times 10^{4}$ & 136 & 9095 & $1.50 \times 10^{-2}$ \\
\hline Philadelphia & $8.10 \times 10^{3}$ & 148.5 & 2690 & $1.80 \times 10^{-2}$ \\
\hline Boston & $2.51 \times 10^{3}$ & 15.0 & 1122 & $1.34 \times 10^{-2}$ \\
\hline Baltimore & $4.88 \times 10^{3}$ & 29.2 & 1854 & $1.57 \times 10^{-2}$ \\
\hline Washington D.C. & $6.98 \times 10^{3}$ & 41.8 & 3087 & $1.35 \times 10^{-2}$ \\
\hline Buffalo & $3.34 \times 10^{3}$ & 20.0 & 1250 & $1.60 \times 10^{-2}$ \\
\hline \multicolumn{5}{|l|}{ U.S.A. (1997.1.12) } \\
\hline Mineapolis & $2.04 \times 10^{4}$ & 122 & 4329 & $2.82 \times 10^{-2}$ \\
\hline St. Louis & $1.55 \times 10^{4}$ & 93.0 & 4061 & $2.29 \times 10^{-2}$ \\
\hline Kansas City & $1.19 \times 10^{4}$ & 71.5 & 4611 & $1.55 \times 10^{-2}$ \\
\hline Las Vegas & $6.35 \times 10^{3}$ & 38.0 & 1552 & $2.45 \times 10^{-2}$ \\
\hline Phoenix & $9.18 \times 10^{3}$ & 55.0 & 4782 & $1.15 \times 10^{-2}$ \\
\hline Tuscon & $2.20 \times 10^{3}$ & 13.2 & 1804 & $7.32 \times 10^{-3}$ \\
\hline \multicolumn{5}{|l|}{ Middle America (1997.2.8) } \\
\hline Mexico City & $9.82 \times 10^{3}$ & 58.8 & 4015 & $1.46 \times 10^{-2}$ \\
\hline Monterrey & $1.63 \times 10^{3}$ & 9.79 & 1701 & $5.76 \times 10^{-3}$ \\
\hline Guadalajara & $2.56 \times 10^{3}$ & 15.3 & 1260 & $1.21 \times 10^{-2}$ \\
\hline Guatemala & $7.23 \times 10^{2}$ & 4.33 & 1184 & $3.66 \times 10^{-3}$ \\
\hline San Salvador & $4.57 \times 10^{2}$ & 2.74 & 1038 & $2.64 \times 10^{-3}$ \\
\hline Tegucigalpa & $3.23 \times 10^{2}$ & 1.93 & 489 & $3.95 \times 10^{-3}$ \\
\hline Managua & $2.75 \times 10^{2}$ & 1.65 & 630 & $2.62 \times 10^{-3}$ \\
\hline San Jose & $8.64 \times 10^{2}$ & 5.17 & 1141 & $4.53 \times 10^{-2}$ \\
\hline Panama & $5.35 \times 10^{2}$ & 3.21 & 891 & $3.60 \times 10^{-3}$ \\
\hline Habana & $3.61 \times 10^{2}$ & 2.16 & 706 & $3.06 \times 10^{-3}$ \\
\hline Kingston & $7.33 \times 10^{2}$ & 4.39 & 891 & $4.93 \times 10^{-3}$ \\
\hline
\end{tabular}


Europe. Ve1n (2997. 2. 3)

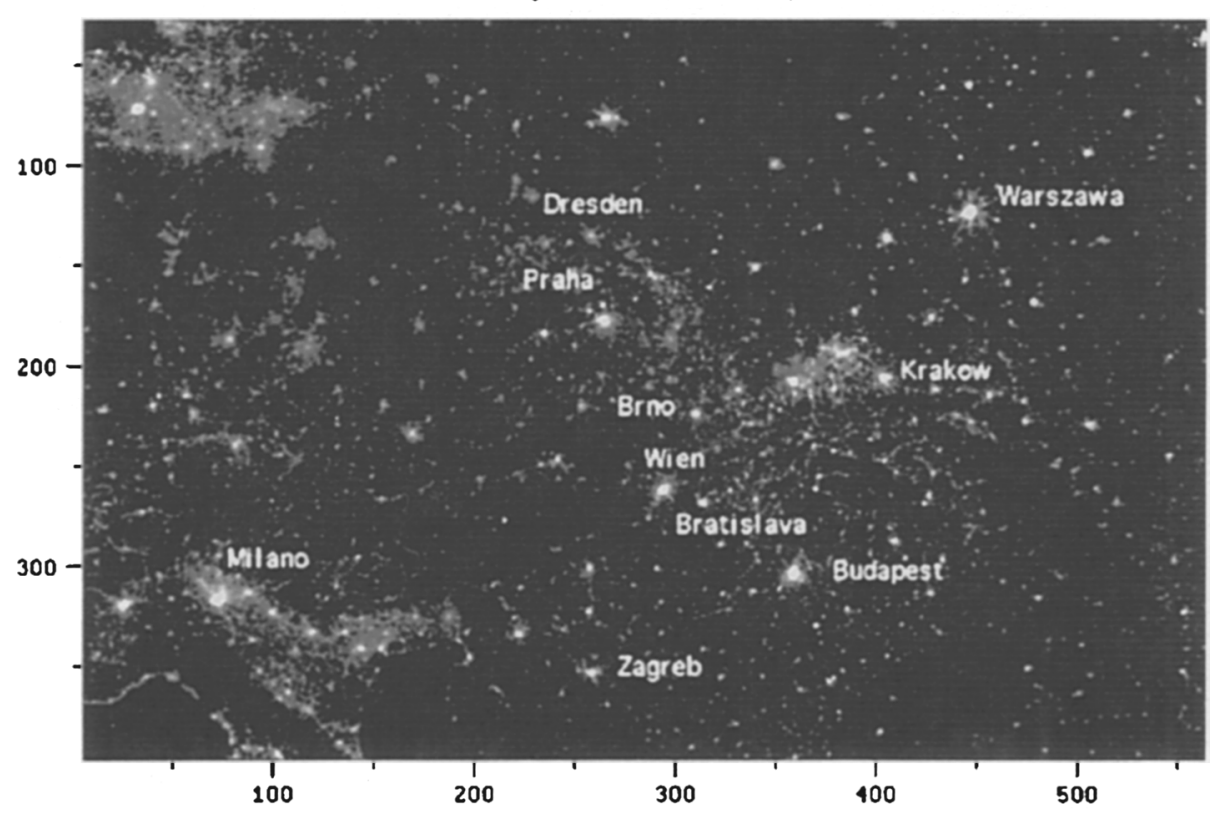

Figure 2. Brightness distribution of Europe on 3 February 1997.

\section{References}

Elvidge, C. D., Baugh, K. E., Dietz, J. B., Bland, T., Sutton, P. C., and Kroehl, H. W. 1999, Radiance Calibration of DMSP-OLS Low-Light Imaging Data of Human Settlements, Remote Sensing and Environment, Vol. 68, $77-88$

Isobe, S. and Hamamura, S. 1998, Ejected City Light of Japan Observed by a Defense Meteorological Satellite Program, ASP Conf. Ser., 139, 191-199

Isobe, S. and Hamamura, S. 2000, Monitoring Light Energy Loss Estimated by the DMSP Satellites, Mem. Soc. Astr. Italiana, 71, 131-138

Kawakami, K. and Isobe, S. 1998, A Study of Luminous Intensity Distribution of Lighting Lamps, ASP Conf. Ser., 139, 161-165

Nakayama, Y. 1992, Poster of Earth at Night

Osman, A., Isobe, S., Nawar, S. and Morcos, A. 2001, this symposium

Sullivan III, W. T. 1991, Earth at Night-An Image of The Nighttime Earth Based on Cloud-free Satellite Photographs, ASP Conf. Ser., 17, 11-17 